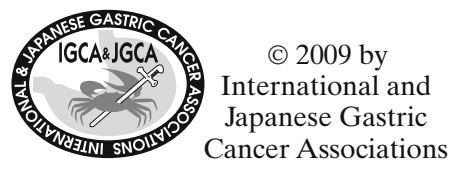

$\underline{\text { Review article }}$

\title{
S-1 review from preclinical pharmacology
}

\author{
Masakazu FuKushima \\ Tokushima Research Center, Taiho Pharmaceutical Co., Ltd., 224-2 Hiraishi-ebisuno, Kawauchi, Tokushima 771-1094, Japan
}

\begin{abstract}
S-1, developed by the scientific theory of both potentiating the antitumor efficacy of 5-fluorouracil (5-FU) and reducing the gastrointestinal (GI) toxicity induced by 5-FU, is a new oral formulation consisting of $1 \mathrm{M}$ tegafur, $0.4 \mathrm{M}$ gimeracil, and $1 \mathrm{M}$ potassium oteracil. By combining gimeracil, a potent inhibitor of 5-FU degradation, and potassium oteracil, which protects against 5-FU-induced GI toxicity to tegafur, S-1, as a dihydropyrimidine dehydrogenase (DPD)-inhibitory fluoropyrimidine (DIF), showed higher antitumor activity, with low intestinal toxicity, compared to continuous infusion of 5-FU (the most effective dosing schedule for 5-FU) and compared to clinically useful oral fluoropyrimidines in various murine and human tumors. In regard to combinations of S-1 with other anticancer drugs, S-1 plus CDDP markedly prolonged survival time in mice suffering gastrointestinal (GI) tumors compared to $S-1$ in combination with mitomycin-C and/or adriamycin. Furthermore, in combination with irinotecan and taxanes (docetaxel), S-1 exercised synergistic antitumor efficacy not only against 5-FU-sensitive GI cancers with low expression levels of thymidylate synthase (TS) but also against 5-FU-resistant GI tumors with originally elevated levels of TS expression. As one of the reasonable mechanisms of anticancer synergism exerted by an S-1 combination, irinotecan and docetaxel were found to downregulate the expression of TS in gastric cancers. Throughout our pharmacological studies of $S-1$, alone and in combination with other anticancer drugs, we found that $S-1$ could be expected to contribute greatly to the treatment of patients with gastric cancer.
\end{abstract}

Key words S-1 · 5-Fluorouracil · Gimeracil · Potassium oteracil · Irinotecan · CDDP · Docetaxel · Thymidylate synthase $\cdot$ Gastrointestinal cancer

Offprint requests to: M. Fukushima

Received: September 16, 2008 / Accepted: October 8, 2008

\section{Introduction}

5-Fluorouracil (5-FU) has been widely used to treat patients with various cancers, such as gastrointestinal, head and neck, and breast cancer, for more than four decades, and its efficacy has been improved by various clinical approaches such as changes of dosing schedules from bolus injection to continuous infusion and the addition of leucovorin (LV). Also, oral formulations of 5-FU as 5-FU prodrugs have been developed to obtain convenient and long-term use in cancer patients. Although 5-FU and its prodrugs are currently administered in combination with other cytotoxic drugs to prolong the survival time of cancer patients, there are unresolved problems with the metabolism of 5-FU and unacceptable toxicities induced by 5-FU, such as gastrointestinal (GI) damage.

5-FU is mainly converted to 5-fluoro-uridine-5'monophosphate by orotate phosphoribosyltransferase (OPRT) and is further metabolized to 5-fluoro2'-deoxyuridine 5'-monophosphate (FdUMP), which tightly binds to thymidylate synthase (TS), a key enzyme in DNA synthesis, with methylene-tetrahydrofolate in tumor, bone marrow, and GI tissues to exert its antitumor activity and toxic effect. However, the majority of 5-FU (about $80 \%$ ) is known to be catabolized to an inactive form by dihydropyrimidine dehydrogenase (DPD) in the liver and tumor tissues. Both strong inhibition of DPD and protection against GI toxicity are therefore needed to potentiate the efficacy of 5-FU without incurring 5-FU-induced severe GI toxicity.

We attempted to develop a novel oral 5-FU formulation which could resolve clinical problems such as those described above. In this review, the characteristics and activities of S-1 alone and in combination with other antitumor drugs used for GI cancer treatment are described. 

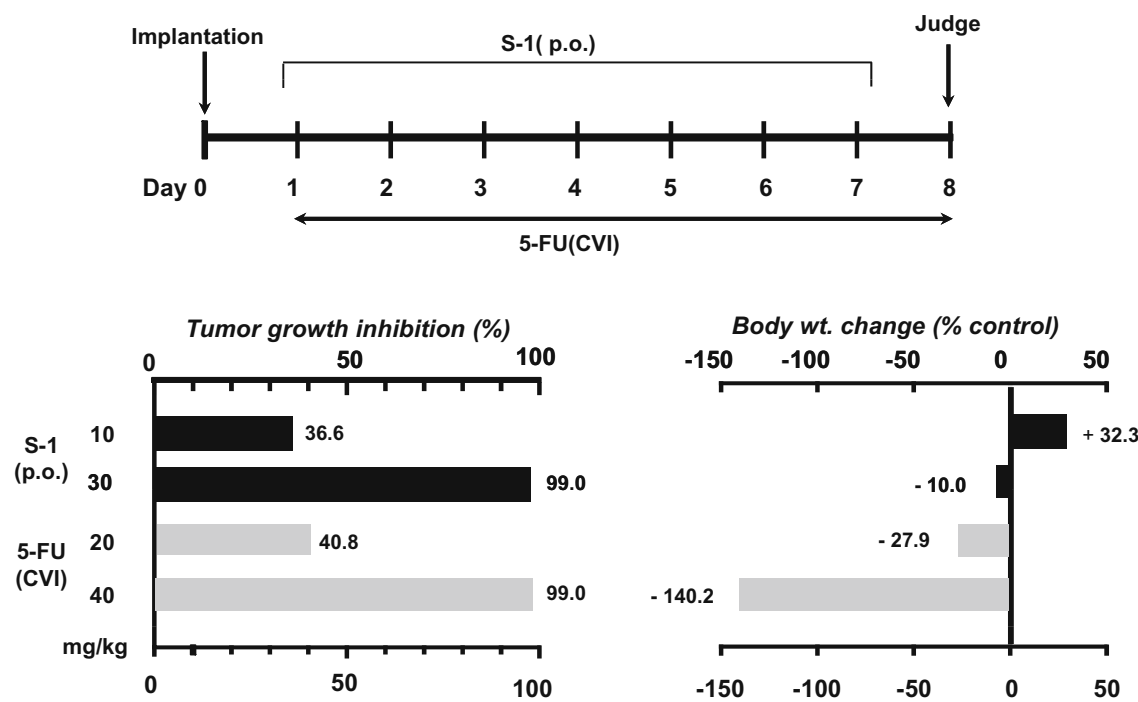

Fig. 1. Antitumor and toxic effects of oral S-1 and infusional 5-fluorouracil (5-FU) in rats with Yoshida sarcomas. Drugs were administered or infused for 7 days, starting $24 \mathrm{~h}$ after the tumor implantation. The antitumor effect and toxicities of oral S-1 and infusional 5-FU were evaluated on day 8. CVI, continuous venous infusion

\section{Preclinical characteristics of S-1}

S-1 is a new oral formulation of 5-FU consisting of $1 \mathrm{M}$ tegafur, 0.4 M 5-chloro-2, 4-dihydroxypyridine (CDHP; gimeracil), and $1 \mathrm{M}$ potassium oxonate (Oxo; potassium oteracil) [1]. The development of S-1 for notable efficacy and lower toxicity in cancer patients was based on the strong inhibition of DPD, leading to high and persistent 5-FU levels in blood and tumors, and the regulation of OPRT in the GI tract, which led to a decrease in GI toxicity, and CDHP [2] and Oxo [3], found by us, enabled the establishment of a new oral 5-FU formulation. When compared with 5-FU infusion, S-1 showed higher antitumor efficacy and lower toxicity in a rodent tumor model, judged by a decrease in the weight of rats, as shown in Fig. 1 [4], suggesting that oral S-1 could contribute to the treatment of cancer patients with higher efficacy and lower GI toxicity compared with infusional 5-FU. The antitumor potency of S-1 also seemed to be greater than that of other oral 5-FU prodrugs in various human cancer xenografts subcutaneously implanted into rodents [5]. Konno et al. [6] and we, independently, evaluated the therapeutic effect of S-1 on human colon carcinoma orthotopically implanted into mice and rats, and found that S-1 significantly inhibited the growth of primary tumors and the metastasis of those tumors to liver [6] or lung [7], suggesting the possibility of clinical benefit to cancer patients.

\section{Combination of S-1 with cisplatin}

Cisplatin (CDDP) is currently most available therapeutic drug to treat patients with gastric, head and neck, and non-small cell lung cancers. Therefore, the combined effect of oral S-1 with CDDP was investigated against rodent and human GI tumors. Kondo and colleagues [8] reported the antitumor activity of daily S-1 combined with high-dose weekly and lower-dose daily CDDP on NUGC-4 tumors in nude mice. We examined whether S-1 plus CDDP achieved the most prolonged survival compared to the combination of S-1 with other anticancer drugs, such as mitomycin-C, irinotecan, and adriamycin in colon 26 PMF-15-bearing mice. As shown in Table 1, S-1 plus CDDP was the most effective combination in prolonging survival in the tumor-bearing mice (unpublished data). These preclinical results suggest that S-1 plus CDDP would be a useful regimen for the treatment of patients with advanced and metastatic gastric cancer.

Two hypotheses for the mechanism of the synergistic anticancer effect exerted by fluoropyrimidine plus CDDP have been advanced, by Shirasaka et al. [9] and Nakano and Niho [10]. One mechanism is an increase in intercellular reduced folate levels induced by CDDP, which potentiates the formation of ternary complex by TS, methylenetetrahydrofolate, and FdUMP derived from 5-FU, and the other mechanism is the cooperative reinforcement of DNA damage in the tumor by 5-FU followed by CDDP.

\section{Combination of S-1 with irinotecan}

Recently, in a number of basic and clinical studies, the expression levels of tumoral TS have been suggested to limit the antitumor efficacy of 5-FU, and we have also confirmed that S-1 showed less antitumor efficacy against human GI cancer xenografts with innate and acquired resistance to 5-FU in which TS was highly expressed [11]. Therefore, the combination of S-1 with other anticancer drugs possessing the ability to 
Table 1. Prolonged survival in mice with advanced metastasis of colon 26 PMF-15 cells in the peritoneal cavity following combined treatment of S-1 with CDDP, MMC, CPT-11, or ADM

\begin{tabular}{lclr}
\hline Drug & Dose $(\mathrm{mg} / \mathrm{kg}$ per day) & \multicolumn{1}{c}{ Treatment } & MST (days) $^{\mathrm{a}}$ \\
\hline Control & - & & 19.5 \\
$\mathrm{~S}-1+$ CDDP & $8.0+12.8$ & Days 1-21, p.o. (S-1) & 38.0 \\
S-1 + MMC & $8.0+2.7$ & Day 1, i.v. (CDDP) & 94.9 \\
S-1 + CPT-11 & $8.0+40.0$ & Days 1-21, p.o. (S-1) & 74.0 \\
S-1 + ADM & $6.7+6.0$ & Days 1,8,15, i.v. (MMC) & 31.5 \\
& & Days 1-21, p.o. (S-1) & 61.5 \\
& & Days 1,8,15, i.v. (CPT-11) & 35.0 \\
\end{tabular}

Each group consists of eight animals

${ }^{a}$ Median survival time

${ }^{\mathrm{b}}$ Increase of life span, ILS $=[($ MST of treatment group $) /($ MST of control group $)-1] \times 100$

Table 2. Effect of CPT-11 administration on the activities of 5-FU-metabolizing enzymes in 4-1-ST, AZ-521, and SC-2 human gastric cancer xenografts in mice

\begin{tabular}{|c|c|c|c|c|c|c|}
\hline \multirow[b]{2}{*}{ Tumor enzyme } & \multicolumn{2}{|c|}{ 4-1-ST } & \multicolumn{2}{|c|}{ AZ-521 } & \multicolumn{2}{|c|}{$\mathrm{SC}-2$} \\
\hline & Control & $\begin{array}{c}\text { CPT-11 } \\
(75 \mathrm{mg} / \mathrm{kg} \text { per } \\
\text { week } \times 2)\end{array}$ & Control & $\begin{array}{c}\text { CPT-11 } \\
(75 \mathrm{mg} / \mathrm{kg} \text { per } \\
\text { week } \times 2)\end{array}$ & Control & $\begin{array}{c}\text { CPT-11 } \\
(75 \mathrm{mg} / \mathrm{kg} \text { per } \\
\text { week } \times 2)\end{array}$ \\
\hline $\mathrm{TS}$ & $2.163 \pm 0.281$ & $1.375 \pm 0.184 * *$ & $0.622 \pm 0.095$ & $0.082 \pm 0.019 * *$ & $0.089 \pm 0.043$ & $0.086 \pm 0.033$ \\
\hline DPD & $2.22 \pm 0.27$ & $1.84 \pm 0.34$ & $78.10 \pm 12.59$ & $53.87 \pm 15.40^{*}$ & $8.580 \pm 3.45$ & $8.120 \pm 1.46$ \\
\hline OPRT & $8.119 \pm 0.663$ & $7.599 \pm 0.806$ & $6.649 \pm 0.772$ & $4.726 \pm 0.600 *$ & $21.01 \pm 2.68$ & $22.10 \pm 4.00$ \\
\hline $\mathrm{TP}$ & $1.011 \pm 0.018$ & $1.168 \pm 0.157$ & $0.115 \pm 0.030$ & $0.103 \pm 0.025$ & $0.647 \pm 0.154$ & $0.676 \pm 0.105$ \\
\hline RNR & $2.030 \pm 0.975$ & $3.076 \pm 1.183$ & $0.483 \pm 0.282$ & $0.660 \pm 0.099$ & $4.330 \pm 1.840$ & $4.150 \pm 0.730$ \\
\hline TK & $15.28 \pm 1.90$ & $15.29 \pm 3.41$ & $31.10 \pm 3.75$ & $35.97 \pm 6.86$ & $32.01 \pm 4.20$ & $68.70 \pm 13.61$ \\
\hline
\end{tabular}

*** Significantly different from nontreated (control) group $(P<0.05$ and $P<0.01$, respectively) by Dunnett's test

CPT-11 $(75 \mathrm{mg} / \mathrm{kg})$ was administered i.v. weekly, twice, to nude mice $(n=5)$ bearing human gastric cancer xenografts, and $24 \mathrm{~h}$ later, tumors were removed and their activities of 5-FU-metabolizing enzymes were measured

Control tumor group received only saline

TS, thymidylate synthase; DPD, dihydropyrimidine dehydrogenase; OPRT, orotate phosphoribosyltransferase; TP, thymidine phosphorylase; RNR, ribonucleotide reductase; TK, thymidine kinase

downregulate highly expressed tumoral TS may be considered to be useful for the treatment of GI cancer patients.

Takiuchi and colleagues [12] reported that irinotecan (which is used clinically to treat gastric and colorectal cancer patients) reduced highly expressed TS in human gastric cancer 4-1-ST and AZ-521, but not SC-2 xenografts, and showed synergistic antitumor activity in combination with S-1 in the 4-1-ST and AZ-521, tumors, while irinotecan plus S-1 therapy was not effective against SC-2 tumors compared with irinotecan and S-1 alone (Table 2; Fig. 2). We further evaluated the antitumor effects of the combination of irinotecan with S-1 against parental and 5-FU-resistant colon cancer KM12C xenografts in mice [13]. As shown in Fig. 3, irinotecan plus S-1 significantly $(P<0.01)$ affected KM12C/5-FU tumors in which S-1 alone showed less antitumor activity, and the activity of irinotecan plus S-1 was similar to that in the parental KM12C tumors. When irinotecan was i.v. administered, the highly expressed TS levels were significantly decreased in KM12C/5-FU tumors, but not in parental KM12C tumors showing low TS activity. In regard to the molecular mechanism of the downregulation of TS by irinotecan in gastric and colon tumors with highly expressed levels of TS, it is suggested that cell cycle-regulating proteins, including CDK4 in the G1 phase of the cell, are regulated by the inhibition of topoisomerase followed by a decrease in $\mathrm{p}-\mathrm{RB}$ and E2F1 proteins, which possibly downregulate the highly expressed levels of TS in the S phase of the cells (Fig. 4). These results reveal that combined therapy with S-1 and irinotecan could be especially useful for the treatment of GI cancer patients with originally high and/or elevated levels of TS. 

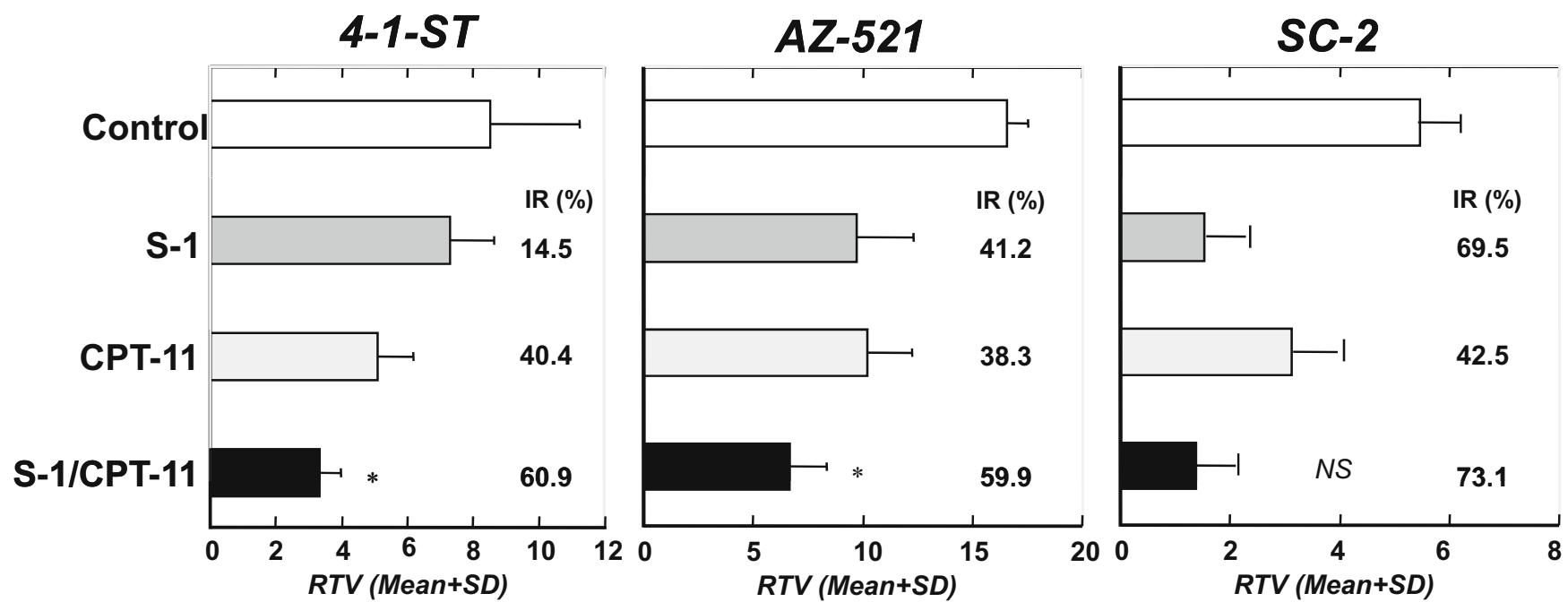

Fig. 2. Antitumor effect of S-1, irinotecan (CPT-11), and their combination on human gastric cancer xenografts in mice. S-1 $(8.3 \mathrm{mg} / \mathrm{kg})$ was orally administered once daily for 14 days, and CPT-11 (40 mg/kg) was injected weekly, twice, to tumor-bearing mice, starting 2 weeks after the tumor implantation. On day 15, the antitumor activity of S-1, CPT-11, and their combination was evaluated as the inhibition rate of tumor growth $(I R ; \%)$. *Significantly different from S-1 alone and CPT-11 alone by intersection union test $(I U T)$. RTV, relative tumor volume

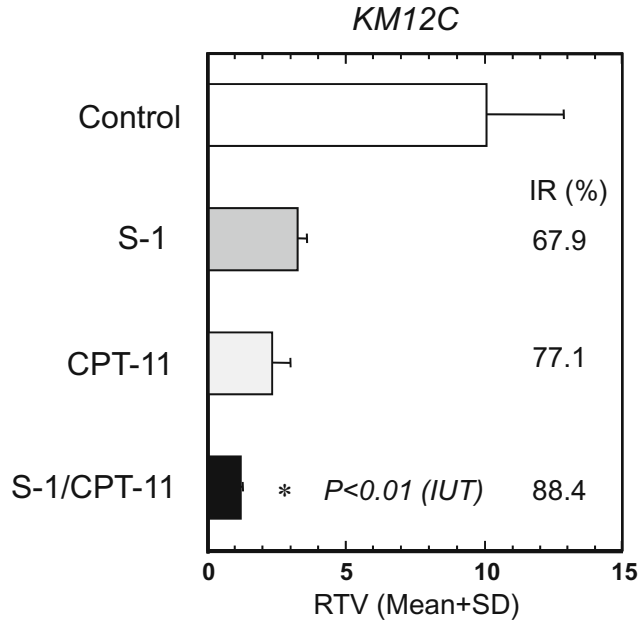

\section{Combination of S-1 with taxanes}

A combination of S-1 with taxanes is thought to be a feasible regimen to treat gastric cancer patients if the taxane biochemically modulates the metabolism or function of 5-FU. For this, Wada et al. [14] investigated the antitumor effects of docetaxel and S-1, using human gastric cancer TMK cells in vitro and in vivo, and they demonstrated that the synergistic antitumor effects of S-1 and docetaxel (as seen in Fig. 5) were based on the modulation of 5-FU-metabolizing enzymes. The expression of TS and DPD significantly decreased and that of OPRT notably increased in docetaxel-treated TMK-1 cells in vitro. In the in vivo tumor models, it is, however, uncertain whether the administration of docetaxel pos- sibly induces a decrease in TS and DPD and an increase in OPRT in TMK-1 tumors. We verified the effect of docetaxel on the activities of 5-FU-metabolizing enzymes in both parental and 5-FU-treated gastric cancer NUGC-3 xenografts in vivo. Weekly administration of docetaxel significantly reduced the activity of TS in the 5-FU-treated tumors, but not in the parental tumors (Table 3), suggesting that combined administration of docetaxel with S-1 could overcome the tumor cell resistance to 5-FU in gastric cancer xenografts with acquired resistance to 5-FU. As seen in Fig. 6, S-1 plus docetaxel showed significantly higher antitumor activity compared to S-1 alone (which showed no antitumor effect) and compared to docetaxel alone against NUGC3/5-FU tumor xenografts. The combination of S-1 with 
docetaxel was significantly more effective than the other two treatments in parental NUGC-3 tumors. An intracellular decrease in cyclin $\mathrm{A}$ and $\mathrm{B}$, and cdc25 proteins induced by docetaxel in the G2/M phase in the cells is a possible mechanism for the downregulation of highly expressed TS in 5-FU-resistant tumors (manuscript in progress for publication).

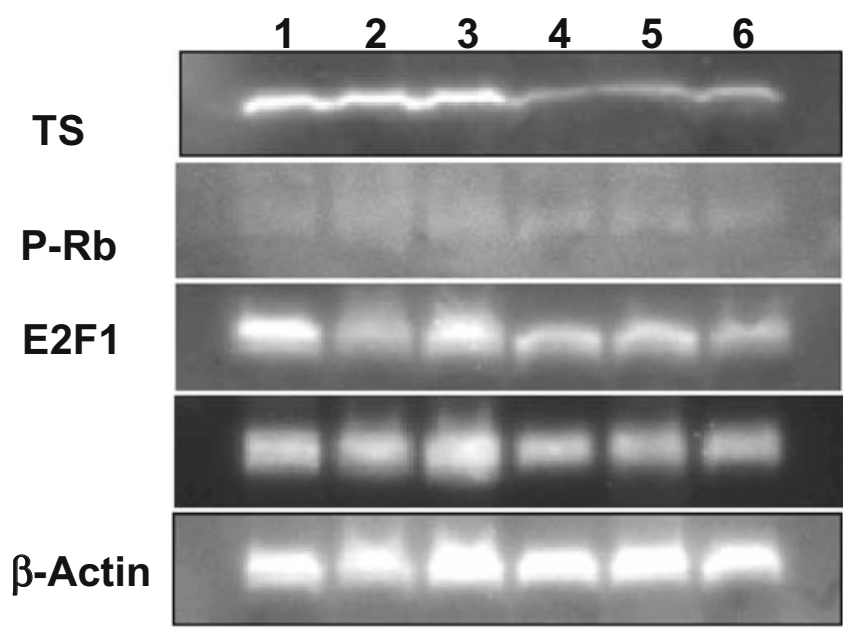

Fig. 4. Expression of thymidylate synthase (TS), pRb, E2F1, and CDK4 proteins in KM12C/5-FU tumors treated with or without CPT-11. CPT-11(75 mg/kg) was administered once weekly for 2 weeks. Tumors were removed 24 hours after, and the expression of these four proteins plus $\beta$-actin was detected by Western-blot methods, using the respective antibodies. Lanes 1 to 3, Control group; lanes 4 to 6, CPT-11-treated group
Throughout these experiments, it appeared that S-1 plus docetaxel could contribute to the treatment of gastric cancer patients in whom S-1 monotherapy or S-1-based combination therapy had failed.

\section{Discussion}

At present, among 5-FU and its oral prodrugs, S-1, as a DPD-inhibitory fluoropyrimidine (DIF), is thought to be the most effective to treat patients with GI cancer. Throughout our preclinical experiments with S-1 alone and/or in combination with CDDP, irinotecan, or docetaxel, these combination therapies showed potential antitumor efficacy against human GI tumor xenografts. Especially, S-1 plus irinotecan and S-1 plus docetaxel showed synergistic antitumor activity in 5FU-resistant GI cancer xenografts with highly elevated levels of TS expression, findings which suggest that alternating or sequential treatment with the three regimens; S-1/CDDP, S-1/irinotecan, and S-1/docetaxel, could prolong survival in cancer patients.

Recently, various molecular targeted agents, including humanized monoclonal antibodies and receptor tyrosine kinase inhibitors, have been developed and introduced to treat GI cancer patients. These drugs exhibit an antitumor effect when combined with classical cytotoxic drugs, and therefore, combinations of S-1-containing regimens with molecular targeted agents would be interesting subjects to further improve the survival of patients with GI cancer.

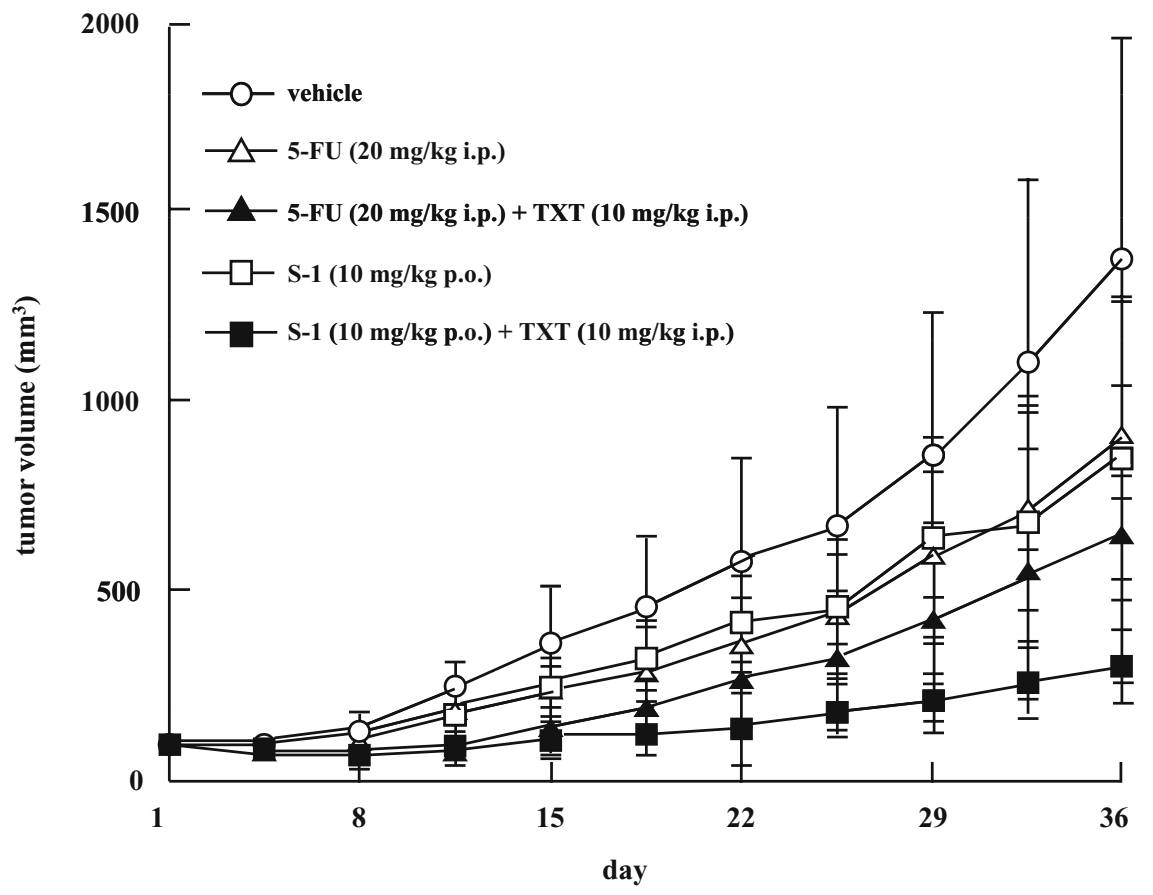

Fig. 5. Comparison of the effects of an S-1/docetaxel $(T X T)$ combination treatment with a 5-FU/TXT cotreatment on the growth of human tumor xenografts. Fragments of TMK-1 tumor tissue were implanted s.c. (bilateral flanks) under anesthesia into nude mice. Tumor growth was established for 7 days after implantation before treatment commenced and the animals were randomly allocated to groups that were treated with vehicle, 5FU $(20 \mathrm{mg} / \mathrm{kg}), 5-\mathrm{FU}$ plus TXT $(10 \mathrm{mg} /$ $\mathrm{kg}), \mathrm{S}-1$ (10 mg/kg), or S-1 plus TXT for an initial 14 days, followed by an additional 14 days after a 1-week interval of no treatment. The data values are expressed as mean (bars, SD) tumor volume 
Table 3. Effect of docetaxel (TXT) on the activities of 5-FU-metabolizing enzymes in parental and 5-FU-resistant gastric cancer (NUGC-3) xenografts

\begin{tabular}{lllll}
\hline & \multicolumn{2}{c}{ NUGC-3 } & & NUGC-3/5-FU \\
\cline { 2 - 3 } Enzyme & \multicolumn{1}{c}{ Control } & \multicolumn{1}{c}{+ TXT } & & Control \\
\hline TS & $0.045 \pm 0.020$ & $0.095 \pm 0.049$ & $1.012 \pm 0.384$ & $0.364 \pm 0.156^{* * *}$ \\
DPD & $11.23 \pm 3.01$ & $13.61 \pm 2.66$ & $15.57 \pm 6.14$ & $13.23 \pm 4.43$ \\
OPRT & $10.08 \pm 1.32$ & $16.24 \pm 11.35$ & $22.75 \pm 7.66$ & $22.72 \pm 7.71$ \\
RNR & $14.79 \pm 7.28$ & $12.34 \pm 3.39$ & $18.75 \pm 4.56$ & $10.81 \pm 2.50$ \\
TP & $1.320 \pm 0.334$ & $1.216 \pm 0.235$ & $0.790 \pm 0.158$ & $1.122 \pm 0.279$ \\
\hline
\end{tabular}

** Significantly different from nontreated (control) group $(P<0.01)$ by Dunnett's test

TXT $(5 \mathrm{mg} / \mathrm{kg})$ was administered i.v. weekly, twice, to tumor-bearing nude mice $(n=5)$, and 24 hours after, tumors were removed and their activities of 5-FU-metabolizing enzymes were measured

Control tumor group received only saline

TS, pmol/mg; DPD, OPRT, and RNR, pmol/mg per min; TP, nmol/mg per min

NUGC-3

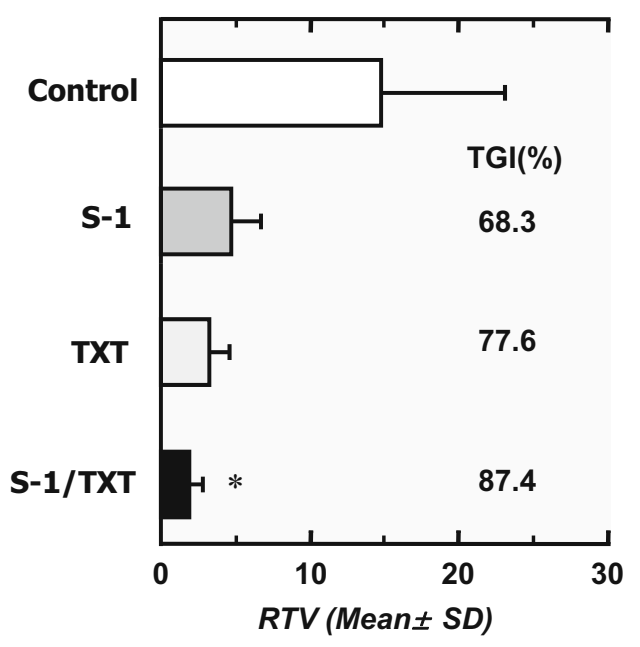

NUGC-3/5-FU

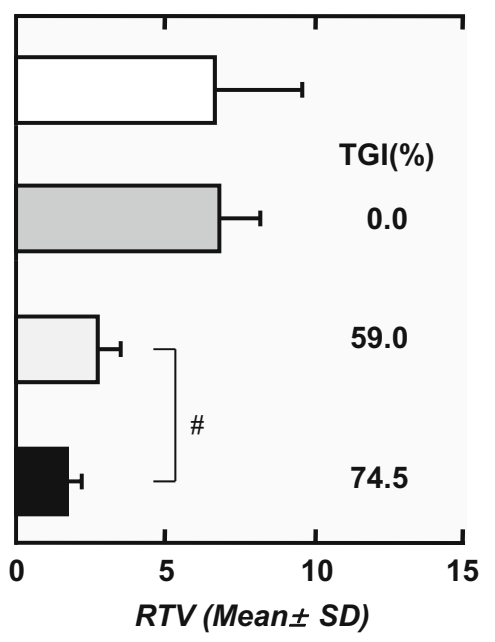

Fig. 6. Antitumor effect of combination chemotherapy with $\mathrm{S}-1$ plus TXT in NUGC-3 and NUGC-3/5-FU human gastric adenocarcinoma xenografts. S-1 $(8.3 \mathrm{mg} / \mathrm{kg})$ was orally administered once daily for 14 days, and TXT $(5 \mathrm{mg} / \mathrm{kg})$ was injected intravenously on days 1 and 8 . Twenty-four hours after, the antitumor activity of each drug and the combination was evaluated as the TGI value *Significantly different from S-1 and TXT alone $(P<0.05$; IUT test $)$; " significantly different from TXT alone $(P=0.0172$; Student's $t$-test)

\section{References}

1. Shirasaka T, Shimamoto Y, Ohshimo H, Yamaguchi M, Kato T, Yonekura K, et al. Development of a novel form of a 5fluorouracil derivative (S-1) directed to the potentiation of the tumor selective cytotoxicity of 5 -fluorouracil by two biochemical modulators. Anticancer Drugs 1996;7:548-57.

2. Tatsumi K, Fukushima M, Shirasaka T, Fujii S. Inhibitory effects of pyrimidine, barbituric acid and pyridine derivatives on 5fluorouracil degradation in rat liver extracts. Gann 1987;78:74855.

3. Shirasaka T, Shimamoto Y, Fukushima M. Inhibition by oxonic acid of gastrointestinal toxicity of 5-fluorouracil without loss of its antitumor activity in rats. Cancer Res 1993;53:4004-9.

4. Fukushima M, Shimamoto Y, Kato T, Uchida J, Yonekura R, Ohshima T, et al. Anticancer activity and toxicity of S-1, an oral combination of tegafur and two biochemical modulators, compared with continuous i.v. infusion of 5-fluorouracil. Anticancer Drugs 1998;9:817-23.

5. Fukushima M, Satake H, Uchidaq J, Shimamoto Y, Kato T, Takechi T, et al. Preclinical efficacy of S-1: a new oral formulation of 5-fluorouracil on human tumor xenografts. Int J Oncol 1998;13: 693-8.
6. Konno H, Tanaka T, Baba M, Kanai T, Matsumoto K, Kamiya $\mathrm{K}$, et al. Therapeutic effect of $1 \mathrm{M}$ tegafur-0.4 M 5-chloro-2,4dihydroxypyridine-1 M potassium oxonate (S-1) on liver metastasis of xenotransplanted human colon carcinoma. Jpn J Cancer Res 1999;90:448-53.

7. Shirasaka T, Nakano K, Takechi T, Satake H, Uchida, Fujioka A, et al. Antitumor activity of $1 \mathrm{M}$ tegafur-0.4 M 5-chloro-2,4dihydroxypyridine-1 $\mathrm{M}$ potassium oxonate (S-1) against human colon carcinoma orthotopically implanted into nude rats. Cancer Res 1996;56:2602-6.

8. Kondo K, Akiyama S, Kasai Y, Kato S, Kuno Y, Kataoka M, et al. Antitumor effect of S-1 and cisplatin treatment against human gastric cancer xenografted in nude mice. Jpn J Cancer Chemother 1997;24:1103-8.

9. Shirasaka T, Shimamoto Y, Ohshima H, Saito H, Fukushima M. Metabolic basis of the synergistic antitumor activities of 5fluorouracil and cisplatin in rodent tumor models in vivo. Cancer Chemother Pharmacol 1993;32:167-72.

10. Nakano S, Niho Y. 5-Fluorouracil and cisplatin sequential chemotherapy; mechanism of action and clinical application in advanced, unresectable non-small cell lung cancer. Intern Med 1995;34:288-91.

11. Fukushima M, Fujioka A, Uchida J, Nakagawa F, Takechi T. Thymidylate synthase (TS) and ribonucleotide reductase (RNR) 
may be involved in acquired resistance to 5-fluorouracil (5-FU) in human cancer xenografts in vivo. Eur J Cancer 2001;37: 1681-7.

12. Takiuchi H, Kawabe S, Gotoh M, Katsu K. Thymidylate synthase gene expression in primary tumors predicts activity of S-1-based chemotherapy for advanced gastric cancer. Gastrointestinal Cancer Res 2007;1:172-7.

13. Fukushima M, Uchida J, Sakamoto K, Ohshimo H, Taguchi T. Molecular mechanism of down-regulation by CPT-11 of thymi- dylate synthase highly expressing in gastrointestinal cancer xenografts during combined treatment with fluoropyrimidines. Eur $\mathrm{J}$ Cancer 2003;Suppl 1:S62.

14. Wada Y, Yoshida K, Suzuki T, Mizuiri H, Konishi K, Ukon K, et al. Synergistic effects of docetaxel and S-1 by modulating the expression of metabolic enzymes for 5-fluorouracil in human gastric cancer cell lines. Int J Cancer 2006;119:783-91. 